\title{
Evaluación de proteínas recombinantes PwCP4, PwCP7 y PwCP9 usando sueros de ratas Wistar infectadas experimentalmente con Paragonimus mexicanus
}

\author{
Luana Gabriele Andrade Gomes ${ }^{1,2, 凶}$, Frank Solano Campos ${ }^{3}$, Gaby Dolz,
}

1 Maestría en Enfermedades Tropicales, PCVET, Universidad Nacional, Heredia, Costa Rica. Email: luagab20@yahoo.com.br, gaby.dolz.wiedner@una.cr

2 Laboratorio de Docencia e Investigación en Medicina Poblacional, Programa MEDPOB, Escuela de Medicina Veterinaria, Universidad Nacional, Heredia, Costa Rica. Email: luagab20@yahoo.com.br, gaby.dolz.wiedner@una.cr

3 Laboratorio de Biotecnología de Plantas, Escuela de Biología, Universidad Nacional, Heredia, Costa Rica. Email: frank.solano.campos@una.cr

La paragonimiasis es una enfermedad zoonótica parasitaria que se considera desatendida y subdiagnosticada en nuestro país. El ser humano puede infectarse a través del consumo de cangrejos de agua dulce crudos o insuficientemente cocidos. Desde 1976 se han reportado 27 casos en Costa Rica, generalmente fueron diagnosticados en niños (90\%) que vivían en zonas rurales (vertiente Atlántica y Pacífica), y estos niños presentaron en un $50 \%$ de los casos enfermedad extrapulmonar. La aplicación de una prueba intradérmica en escolares de Talamanca en el año 1999 determinó un 23 $\%(23 / 100)$ de los niños con reacciones positivas a Paragonimus mexicanus. Otro estudio realizado en el 2015 estableció un alto consumo de cangrejo de agua dulce (21\%) en la población, y determinó un 12.7 \% (44/347) de cangrejos de 67 \% (8/12) sitios analizados como infectados con el parásito. Debido a que no existe prueba diagnóstica inmunológica para determinar la presencia de paragonimiasis extrapulmonar en la población humana de Costa Rica, el objetivo del presente estudio fue obtener y caracterizar diferentes proteínas recombinantes del parásito, para ser utilizadas como antígenos en una prueba inmunoenzimática. Se expresaron y purificaron diferentes cisteínas proteasas (CP) recombinantes de Paragonimus westermani ( $\mathrm{Pw}$ ), las cuales, posteriormente, se evaluaron mediante la técnica de immunoblot con sueros de ratas Wistar infectadas experimentalmente con $P$. mexicanus y ratas Wistar controles (no infectadas con P. mexicanus). Los tres antígenos recombinantes (PwCP4, PwCP7 y PwCP9) resultaron ser específicos, ya que reconocieron los anticuerpos presentes en el suero de ratas infectadas, no así en las ratas no infectadas. Se concluye, que estas proteínas parecen ser promisorias para ser utilizadas en el diagnóstico inmunológico de la parasitosis.

Autor para correspondencia Luana Gabriele Andrade Gomes: luagab20@yahoo.com.br 